\title{
Development of Mission Adaptive Digital Composite Aerostructure Technologies (MADCAT)
}

\author{
Kenneth Cheung ${ }^{1}$, Daniel Cellucci ${ }^{2}$, Grace Copplestone, Nick Cramer, Jesse Fusco, Benjamin \\ Jenett $^{3}$, Joseph Kim, Alexandria Langford, Alex Mazhari, Greenfield Trinh, Sean Swei \\ NASA Ames Research Center, Moffett Field, CA, 94035
}

\begin{abstract}
This paper reviews the development of the Mission Adaptive Digital Composite Aerostructures Technologies (MADCAT) v0 demonstrator aircraft, utilizing a novel aerostructure concept that combines advanced composite materials manufacturing and fabrication technologies with a discrete construction approach to achieve high stiffness-todensity ratio ultra-light aerostructures that provide versatility and adaptability. This revolutionary aerostructure concept has the potential to change how future air vehicles are designed, built, and flown, with dramatic reductions in weight and manufacturing complexity - the number of types of structural components needed to build air vehicles - while enabling new mission objectives. We utilize the innovative digital composite materials and discrete construction technologies to demonstrate the feasibility of the proposed aerostructure concept, by building and testing a scaled prototype UAV, MADCAT v0. This paper presents an overview of the design and development of the MADCAT v0 flight demonstrator.
\end{abstract}

\section{Introduction}

In response to future aviation needs and challenges, NASA has defined a set of aggressive fuel efficiency metrics for $\mathrm{N}+2$ and $\mathrm{N}+3$ aircraft [1]. The study of "economically practical approaches to improve aircraft efficiency and minimize environmental impact" is of great significance to associated aeronautical challenges. A common thread in the requirements for viable solutions to meet these challenges is the need for lightweight structural concepts. Typical solutions for weight reduction involve the use of high stiffness-to-weight ratio materials that are typically used to enable the construction of thinner and lighter structures capable of sustaining the required ultimate loads. In doing so, however, challenges related to practical considerations during flight arise; these include aerodynamic stability and adverse consequences from aeroelastic interactions with aerodynamic forces and moments. The research presented in this paper involves the use of a combination of lightweight materials and lattice based cellular components to construct wing aerostructures $[2,3]$. This provides a strategy for lightweight structures with precisely tunable flexibility to allow reconfigurations of control actuation to achieve optimal aerodynamic efficiency. This proposed concept thus constitutes an innovative

\footnotetext{
1 Corresponding Author: kenny@nasa.gov

2 Graduate Student, Cornell University, NASA Space Technology Research Fellow

${ }^{3}$ Graduate Student, MIT, NASA Space Technology Research Fellow
} 
approach to address both structural and aerodynamic efficiency with an integrated lightweight aerostructure platform. Rigorous testing of a lattice based composite vehicle to determine operational feasibility of a stable and continuously deformable wing has not been previously studied in great depth.

Earlier research studies conducted by NASA support the claims that active control of wing twist and deflection at local wing sections can improve overall aerodynamic efficiency $[4,5,6]$. Though the induced aerodynamic drag improvements of the benchmark wing configuration with the drooped wing and the inflected wing [6]; see Fig. 1, are significant (overall induced drag reduction of $5.3 \%$ and $3.5 \%$, respectively), in order to ensure the optimal drag reduction in actual flight conditions, active wing shaping controls are desired. Two recent NASA projects to realize in-flight aeroelastic wing shape control are the Variable Camber-Continuous Trailing Edge Flap (VCCTEF) and Distributed Electric Propulsion (DEP) concepts. The VCCTEF strategy divides a trailing edge into many flap sections, each of which can be individually controlled [6], within the constraints of an interstitial elastomeric interpolating surface. Chord-wise and span-wise adaptive wing shaping for various flight scenarios can be achieved in this manner [7]. DEP proposes to accomplish similar shape morphing modes, but with differential control of spatially distributed electric propulsion systems. The weight penalty and system challenges associated with the need for many very powerful VCCTEFs or DEP systems lead us to seek lighter weight architectures. Instead of performing primary deformation modes (i.e. wing twist) as elastic reactions that are coupled to distributed flap/propulsion systems, we propose to revisit direct internal actuation with a novel distributed structural compliance strategy. A good account of wing twist/morphing mechanism discussion can be found in [8] and the references therein.

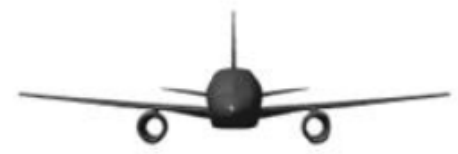

Benchmark

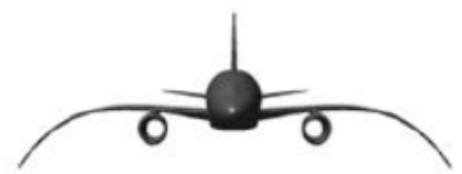

Drooped Wing

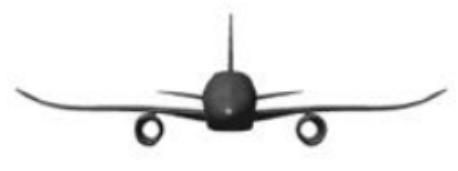

Inflected Wing

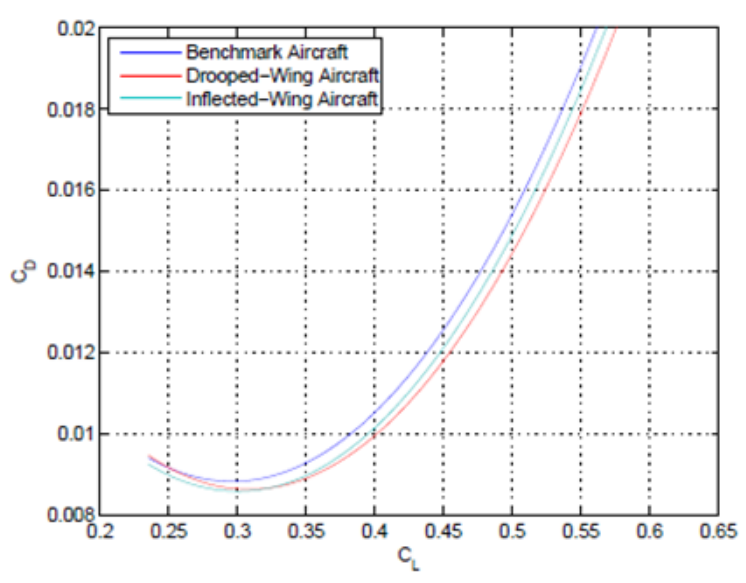

Figure 1: Drag polar comparisons: benchmark, drooped wing, and inflected wing [6] 
This paper presents a novel active wing morphing aerostructure concept by combining the advanced composite lattice-based cellular materials/components and the multi-objective optimal flight control design to realize continuous morphing wing control, so as to improve aerodynamic efficiency for air vehicles. In particular, this concept leverages a building block strategy for lattice-based components that provide great adaptability to varying flight scenarios through inflight wing shaping control. Unlike conventional aircraft control actuation mechanisms, with rigid kinematic flaps and electric/hydraulic motor driven components, the lattice-based cellular wing structure provides the necessary deformation compliance through the actuation of heterogeneous structural elements along the span-wise direction to achieve a desired wing shape according to real time flight conditions.

In this paper, we provide a high-level overview of the development of a scaled prototype UAV demonstrator, MADCAT v0, and highlight some of the salient features of the vehicle.

\section{Latticed-based Wing Structures}

Figure 2 highlights the construction process for a pair of lattice-based composite flexible wings, based on the conventional NACA 0012 airfoil profile with $1 \mathrm{ft}$ chord length. Further details of the manufacturing process can be found in [8]. According to the expected global behavior and loading condition, different airfoil sections are filled with different lattice geometries to create different density and load response behavior. For instance, near the quarterchord where stretch load is dominant and the trailing edge where bending is dominant [8].
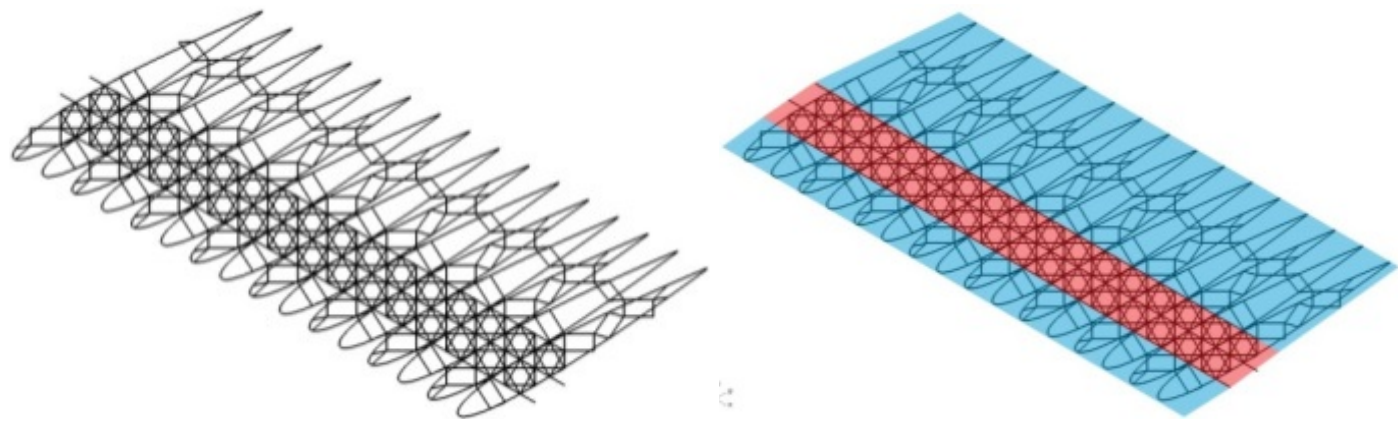

Figure 2: A lattice-based construction of wing structures - varying density

\section{A. Bench Testing and Model Validation}

A simulation wing model is developed and validated using both bending and torsional test data. Images of the three-point bending experiment are provided in [8]. The nodes on both ends of the wing were constrained vertically, and a vertical load was applied to nodes along the central cell boundary. The simulations predicted a flexural modulus of $184 \mathrm{MPa}$, which is very close to the experimental result of $191 \mathrm{MPa}$. Images of the torsional loading test setup are provided in [8]. The nodes at one end of the wing were constrained, and a vertical force was 
applied to the other end at the trailing edge. The axis of rotation is assumed to be near at quarterchord. The simulations predicted a torsional spring constant of $14.9 \mathrm{Nm} /$ radian in the linear range. The experimental result is $12 \mathrm{Nm}$ /radian.

\section{B. Actuation Mechanism}

To actuate the span-wise wing twist, we rotate the wing through the use of a carbon fiber rod that is fixed to an end plate, as shown in Fig. 3a. The servos used for the actuation are placed in the fuselage, and they are connected to the carbon fiber rods through the custom 3D-printed servo mounts/arms; see Fig. $3 \mathrm{~b}$.

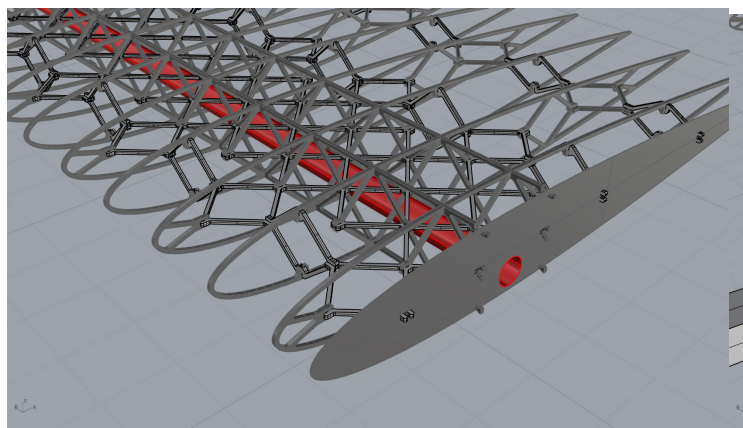

(a)

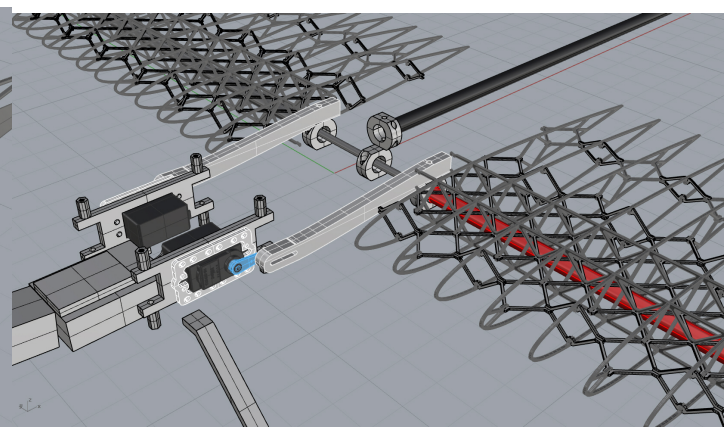

(b)

Figure 3: (a) End piece connection (carbon fiber rod is shown in red); (b) actuation assembly.

Therefore, the wing twist actuation is achieved through a flexural arm, as illustrated in Fig. 4. A favorable torque ratio of 5:1 is attained through the linkage geometry. It should be noted that the axis of rotation is near the quarter-chord.
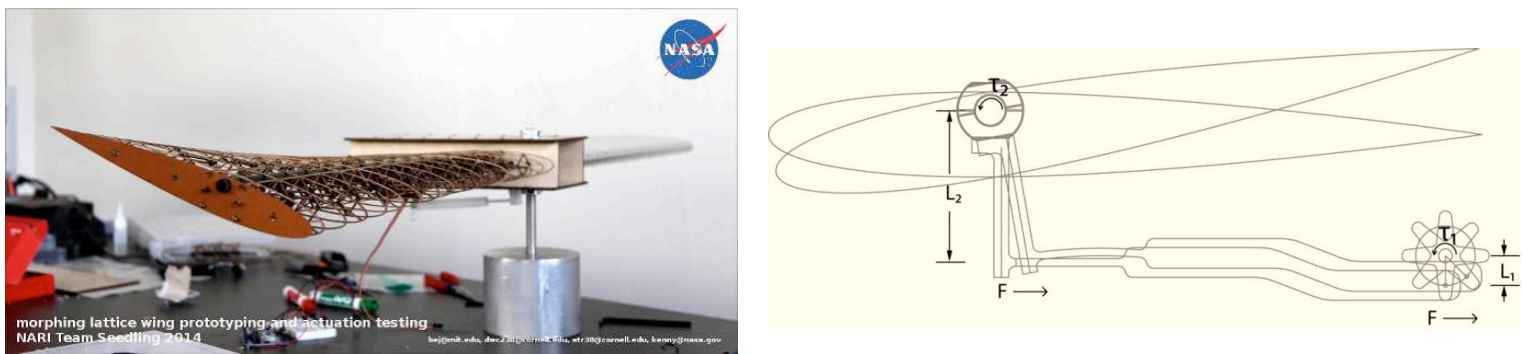

Figure 4: Wingtip twist mechanism

\section{Lattice Structure Modeling and Control}

The discrete or "building block" construction of lattice-based aerostructure introduces an independent design degree of freedom that allows global functional properties to be determined by the local component behavior. As demonstrated earlier, the lattice structures can be assembled from many discrete parts, for which there is a small number of distinctive part types. The discrete construction of these materials will then allow for combinatorial optimization of system designs. These benefits make the lattice-based aerostructure an ideal candidate for the design and manufacturing of morphing wings. Figure 5 shows a section of flexible wing considered earlier, 
and it naturally presents itself as a series of lumped mass model. As shown, each rib of the wing section provides a logical location for a lumped mass, and the connecting components between each airfoil can be modeled as spring and damper. Furthermore, the bench test data given in Section II.A can be used for modeling the flexible connectors.

By utilizing the extended discrete-time transfer matrix method (E-DT-TMM) [9], a reduced order model for mass $n$ can be described by the following discrete time state-space representation,

$$
X_{n}(k+1)=A_{n n} X_{n}(k)+B_{n}\left[\gamma(k)+f_{n}(k)\right]
$$

where $\boldsymbol{k} \geq 0$ is the time step, $\boldsymbol{X}_{\boldsymbol{n}}$ denotes the states of mass $n, \boldsymbol{A}_{n \boldsymbol{n}}, \boldsymbol{B}_{\boldsymbol{n}}$ are constant matrices, $\gamma(\boldsymbol{k})$ denotes the exogenous input, and $\boldsymbol{f}_{\boldsymbol{n}}(\boldsymbol{k})$ is the control input applied at mass $n$. A globally stabilizing controller can be derived from a collection of decentralized controllers designed for each actuated mass. Detail derivations can be found in Section 4 of [9].

\section{Wind Tunnel Test}

To assess the flight performance of the designed lattice-based wing, a series of wind tunnel tests were performed at various configurations. Aerodynamic results and details of these tests can be found in [12]. As indicated, the flexible lattice wing model is torque tube actuated to produce twist motion. The angle of attack $(\alpha)$ sweep was performed between -4 and +16 degrees with increments of 2 degrees, and the sideslip angle $(\beta)$ sweep at $(-4,0,+4)$ degree. These tests were conducted at dynamic pressure up to $7 \mathrm{psf}(\sim 50 \mathrm{mph})$, with static wing tip twist ranging from 6 degrees trailing edge-up to 10 degrees trailing edge-down. Additional tests were conducted with wing tip twist oscillation at various frequencies, ranging from $0.5 \mathrm{~Hz}$ to $5.4 \mathrm{~Hz}$, and at various dynamic pressures. Forced roll oscillation tests were also conducted at $0.5 \mathrm{~Hz}$ and $1 \mathrm{~Hz}$, both synchronously and asynchronously, to assess roll/yaw performance.

For comparison purpose, a similar matrix of conditions was tested using a rigid model with identical (neutral) outer model line geometry, as shown in [12]. Flaperons were included on the rigid model to represent typical symmetric and differential control surfaces for comparisons to the flexible lattice model undergoing commanded twist deflections.

A series of static tests were performed with varying angles of attack, followed by symmetric flaperon deflection angles (labeled "flap" in the figures) or tip twist in the case of the flexible model, Figs. 10 and 11. It can be seen that "10-deg Flap" curve (a) is very close to "6-deg TipTwist" curve (b), suggesting that there is a proportionality between the control effectiveness of the two models.

The key findings from the wind tunnel tests can be summarized as follows: 1) the maximum aero load was about $21.5 \mathrm{lbf}$; 2 ) the flexible wing model easily withstood aero loading across typical UAV flight envelope, i.e. dynamic pressure up to $7 \mathrm{psf}(10 \mathrm{~Pa})$, speed up to $50 \mathrm{mph}$, thru post-stall angle of attack $\left(>16^{\circ}\right)$, and moderate sideslip angles (generally only $\left.\pm 4^{\circ}\right) ; 3$ ) the flexible wing model at neutral twist exhibited similar aero properties as rigid variant in 
performance and static stability and roll-damping; 4) the flexible wing model allows for improved control options to enhance efficiency as compared to conventional design; 5) Controlsactive tests demonstrated viability of active twist response dynamics against realistic loads and states.

\section{Development of MADCAT v0 Prototype}

To further investigate the performance of the proposed lattice-based active twist wing concept, a scaled prototype MADCAT v0 UAV is developed by utilizing the same pair of twist wings that were wind tunnel tested, as discussed in Section II. Figure 5 shows the CAD prototype for MADCAT v0, and some basic specifications are listed in Table 1. The detail design and development studies can be found in [10].

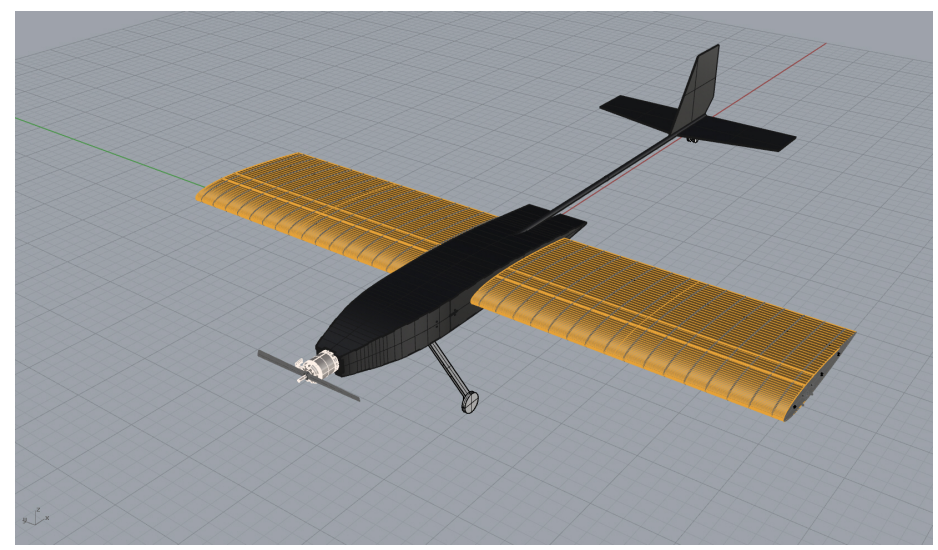

Figure 5: MADCAT 3D configuration

Table 1: MADCAT v0 specifications

\begin{tabular}{|c|c|c|c|}
\hline Weight (lb) & Wingspan (in) & Length (in) & Aspect Ratio \\
\hline$<10$ (include HW) & 52 & 56 & 3.81 \\
\hline
\end{tabular}

\section{A. Fuselage Design}

The design followed the modular approach, by binding individual flat panels together through zip ties. Zip ties are critical part of rapid prototyping; when properly tensioned, zip ties are structurally comparable to double-shear bolts. In order to accommodate two servo motors that are connected to vertical moment arm to generate the twist momentum, the width and height of the fuselage were chosen to be 5.5 in and 2.25 in, respectively. The height was further tapered down to resemble a symmetrical airfoil, and the fuselage is 24 in long. The final fuselage was manufactured with a water-jet from a customized carbon fiber composite laminate; see Fig. 6. 


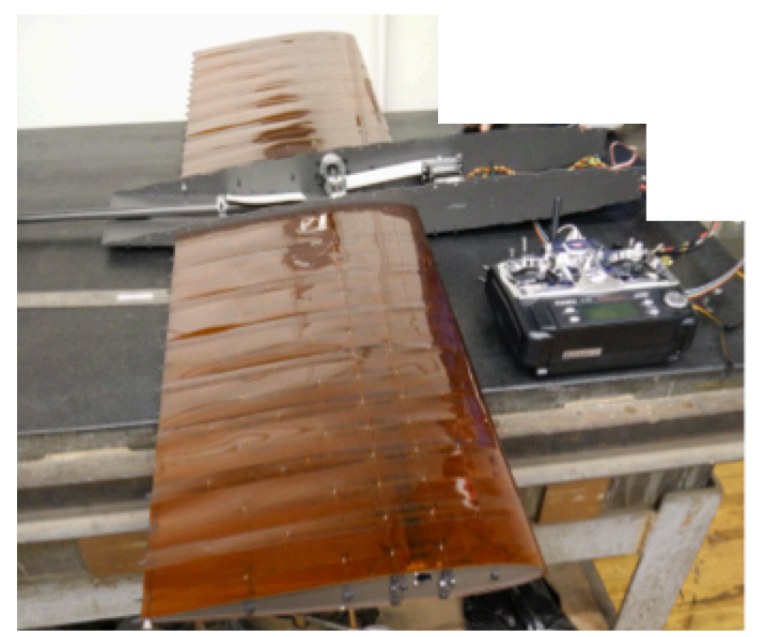

Figure 6: Carbon fiber fuselage assembling with wings

\section{B. Empennage Design}

The empennage is configured in a conventional format, with a tapered vertical stabilizer placed at the half span of a symmetrical horizontal stabilizer, as shown in Fig. 7. The vertical stabilizer and the rudder have a surface area of $64 \mathrm{in}^{2}$ and $14.7 \mathrm{in}^{2}$, respectively, and the rudder is integrated with a servo producing $+/-30$ deg deflection. The horizontal stabilizer and the elevator have a surface area of $110 \mathrm{in}^{2}$ and $40 \mathrm{in}^{2}$, respectively. Furthermore, the elevator is actuated through a servo and able to deflect $+/-30 \mathrm{deg}$.

The horizontal tail volume coefficient is determined to be 0.534 , which is well within the range of balanced aircraft, while the vertical tail volume coefficient is 0.081 , which indicates a sufficient directional control authority. A 0.5 in diameter and 36 in long carbon fiber rod, with a thickness of $0.1 \mathrm{in}$, is integrated as the moment arm connecting the stabilizers and the fuselage.

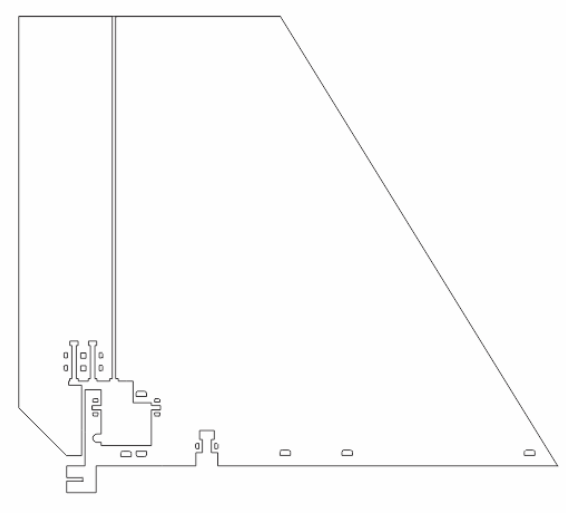

(a)

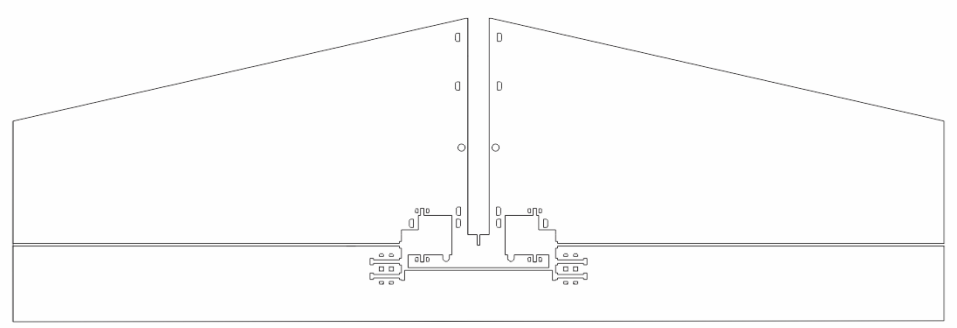

(b)

Figure 7: (a) Vertical stabilizer and rudder; (b) horizontal stabilizer and elevator 


\section{Propulsion System}

The propulsion system is optimized for high thrust efficiency in terms of grams per watt. Large excess power reservoir is a key design factor for an experimental UAV, as it allows the pilot to counter any unforeseen and adverse aerodynamic effects. A suite of propulsion components consisted of a brushless motor, 3-cell lithium ion battery, an electronic speed controller (ESC), and a propeller. The chosen propulsion system generates $248 \mathrm{~W}$ by revolving the propeller at 7,800 cycles per minute, which is equivalent to a current draw of $22.4 \mathrm{amps}$ and 60 ounces of thrust. Under the current configuration, a maximum current draw of 22.4 amps from the motor and 6 amps draw from ESC and actuation systems requires the total steady current of 28.4 amps. With the battery rating at 5.0 amps hour capacity, the propulsion system provides about 11 minutes of flight time under full load.

A receiver with relative high voltage capacity was selected. When paired with a transmitter, the aircraft was able to be wirelessly controlled in both throttle and control signal input. The configuration of the implemented system is shown in Fig. 8.

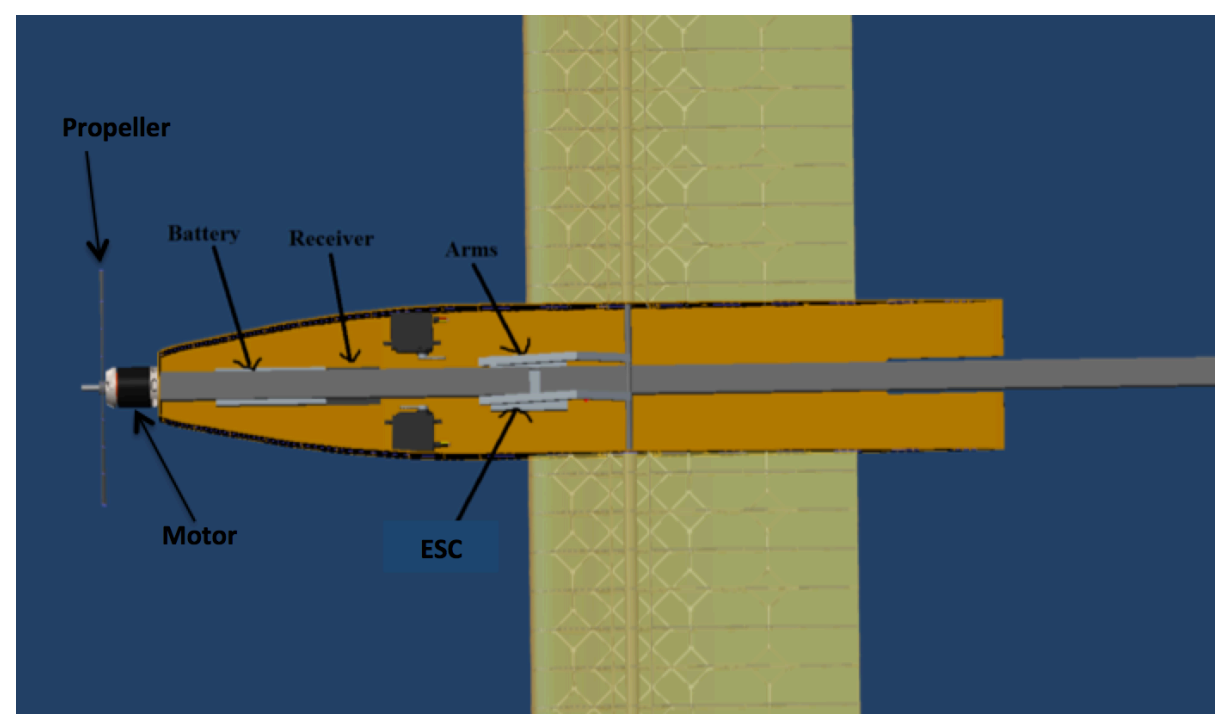

Figure 8: A suite of propulsion components

\section{MADCAT v0 Instrumented Flight Test}

The MADCAT v0 is designed to replicate the roll generation of the traditional ailerons with an active twist mechanism. As was demonstrated in the wind tunnel testing, there was an effective proportionality between the conventional ailerons and the active twist. The viability of the active twist mechanism would need to be flight tested to assess its twist control authority and efficiency. 


\section{A. Instrumentation Hardware Development}

The hardware system for MADCAT v0 is to enable the active wing twist and assess its performance. It is also aimed to allow the flight system identification suite to operate completely independently from the flight critical components. The basic configuration of the avionics is shown in Fig. 9, where the actuation system is highlighted in the orange box, the receiver hardware is colored in light green, while the power components are in the darker green, and the sensors are in yellow.

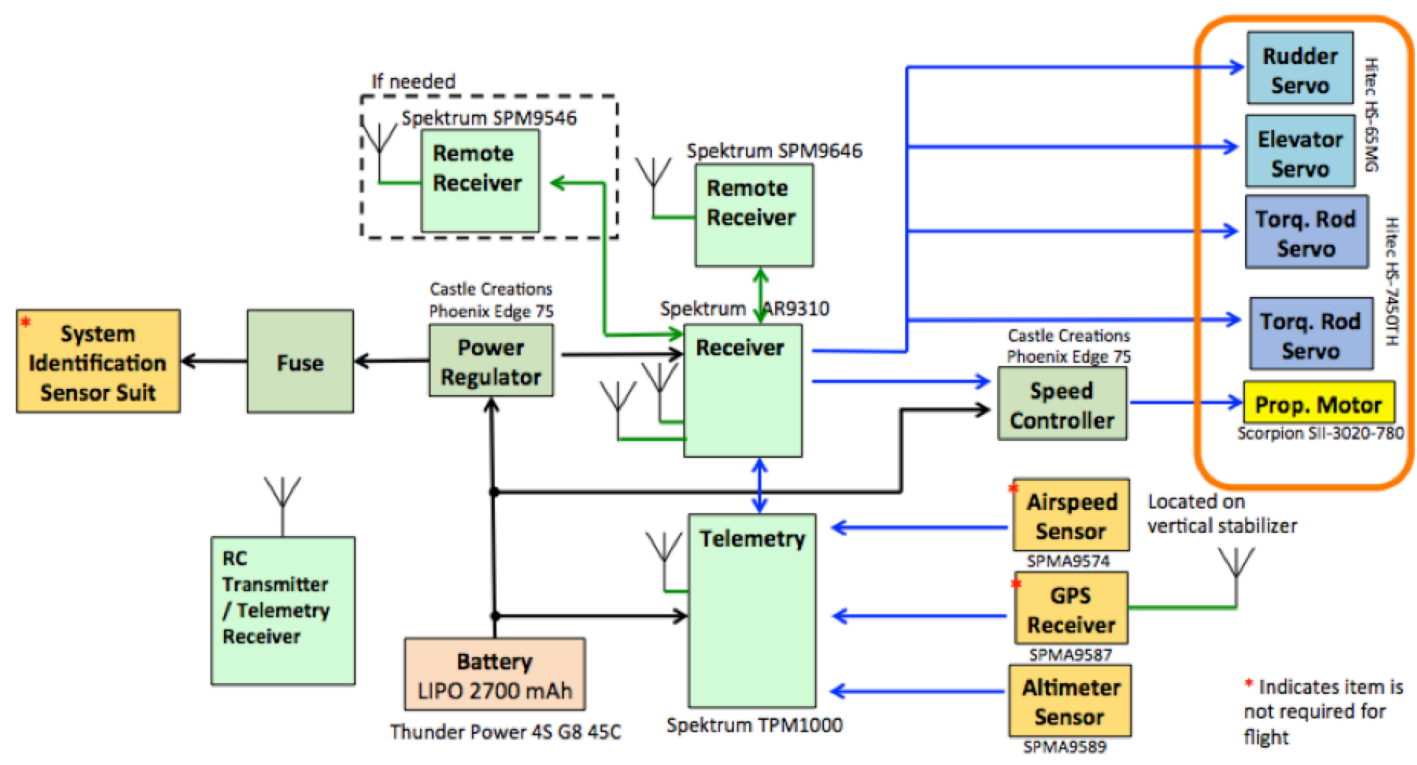

Figure 9: MADCAT v0 flight control architecture

Figure 10 shows a detailed breakdown of the system identification sensor suite, which includes a data acquisition system, potentiometers and distributed IMUs. The IMUs are distributed along the wing span and in the fuselage, as shown in Fig. 11, where an IMU is placed on both the leading and trailing edges, at the wing tip, at the three quarter wing length, and at the half wing length. In addition, there are string potentiometers attached to all of the control surfaces for measuring the deflections, see Fig. 12. All sensor data are collected by a BeagleBone Black with a custom cape acting as a data acquisition device. 


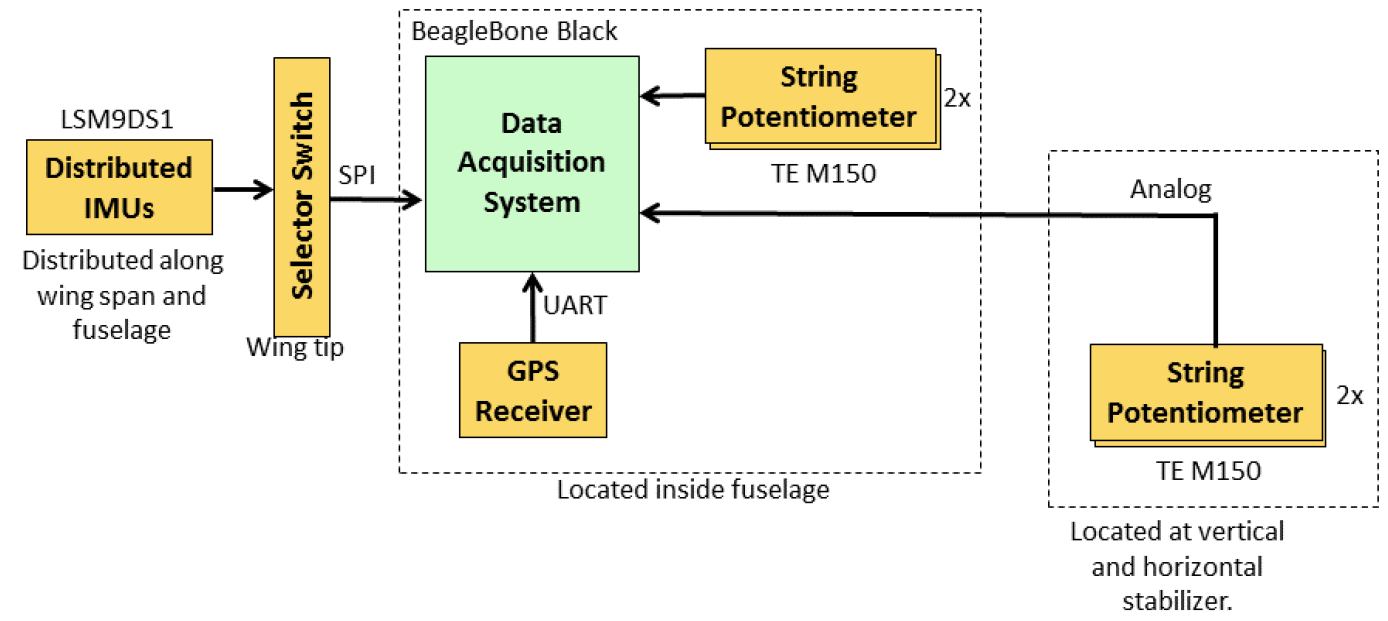

Figure 10: System identification sensor suite configuration

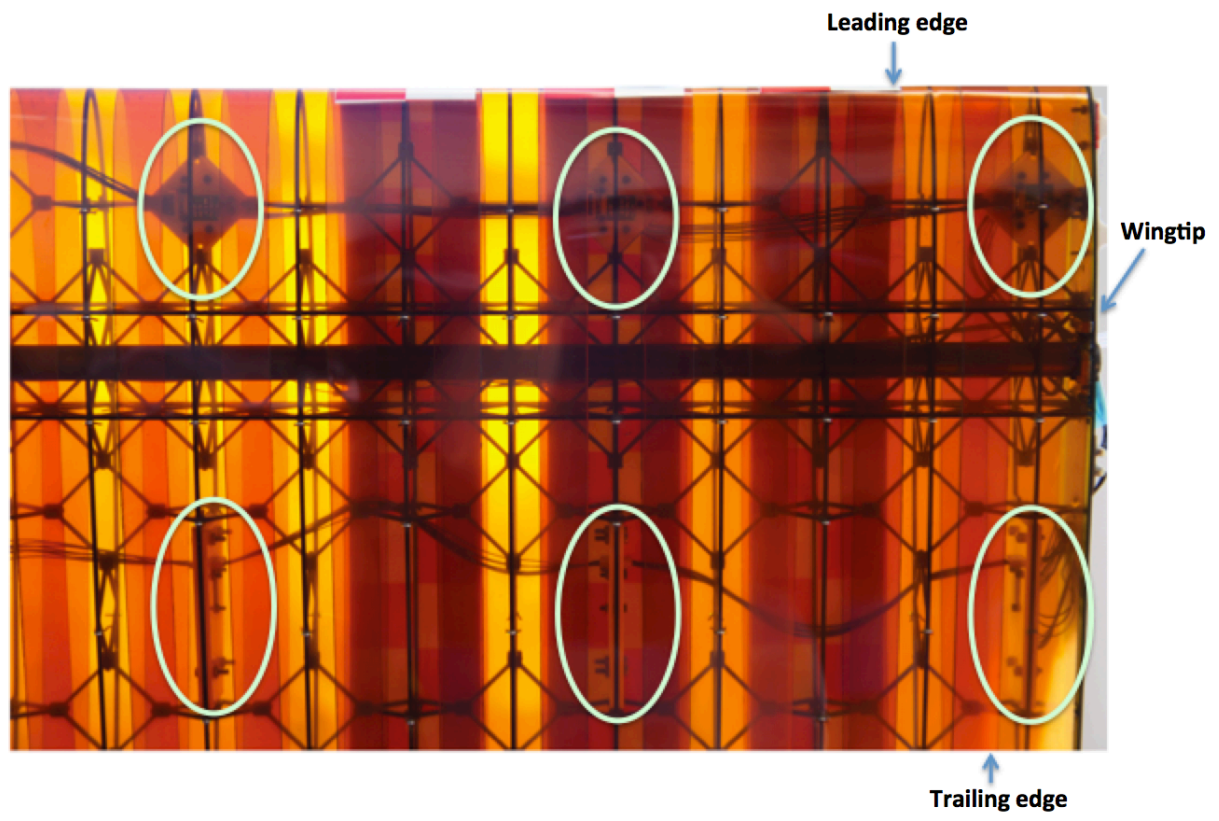

Figure 11: MADCAT v0 Wing embedded IMUs 


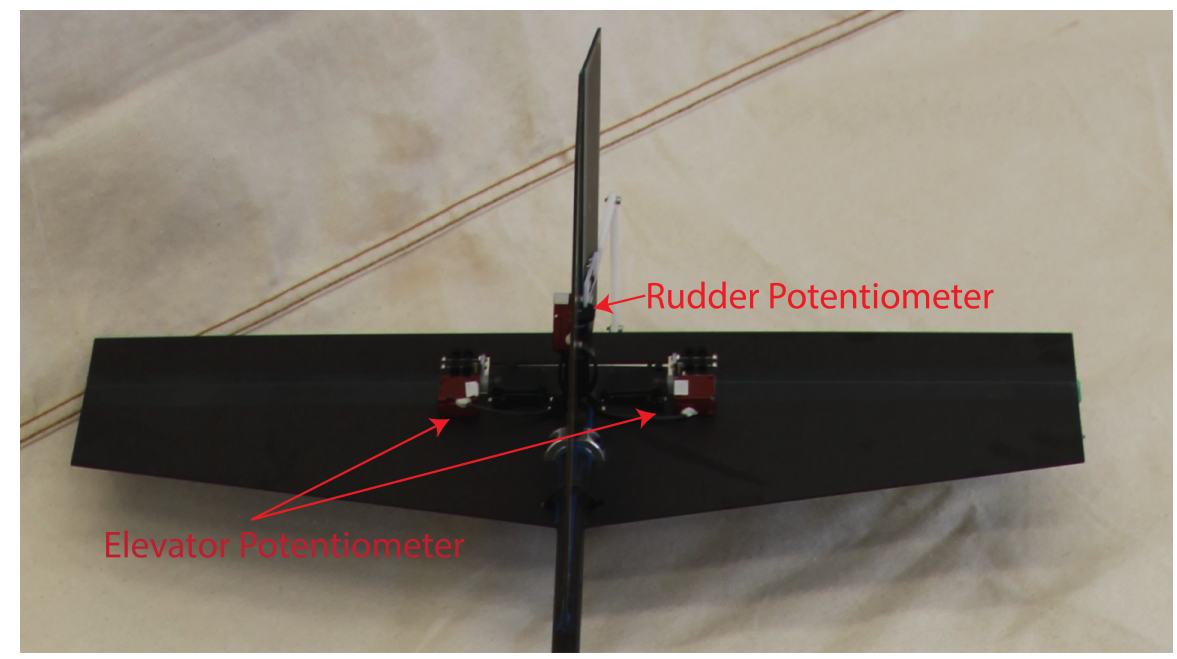

Figure 12: String potentiometers to measure the rudder and elevator deflection

\section{B. Instrumented Flight Test}

The instrumented flight tests were performed at the NASA Crows Landing Flight Test Facility; see Fig. 13. Four flight tests were conducted, which included the pre-flight test for pilots to familiarize with MADCAT v0, the frequency sweeps in roll, pitch, and yaw maneuvers, and the assessment of stall dynamics. The frequency sweeps were performed by the pilot oscillating the control stick from $1 \mathrm{~Hz}$ to about $5 \mathrm{~Hz}$ with respect to each control axis. All the flight test images were also recorded via a 360-deg video camera mounted on top of the fuselage, as shown in Fig. 14.

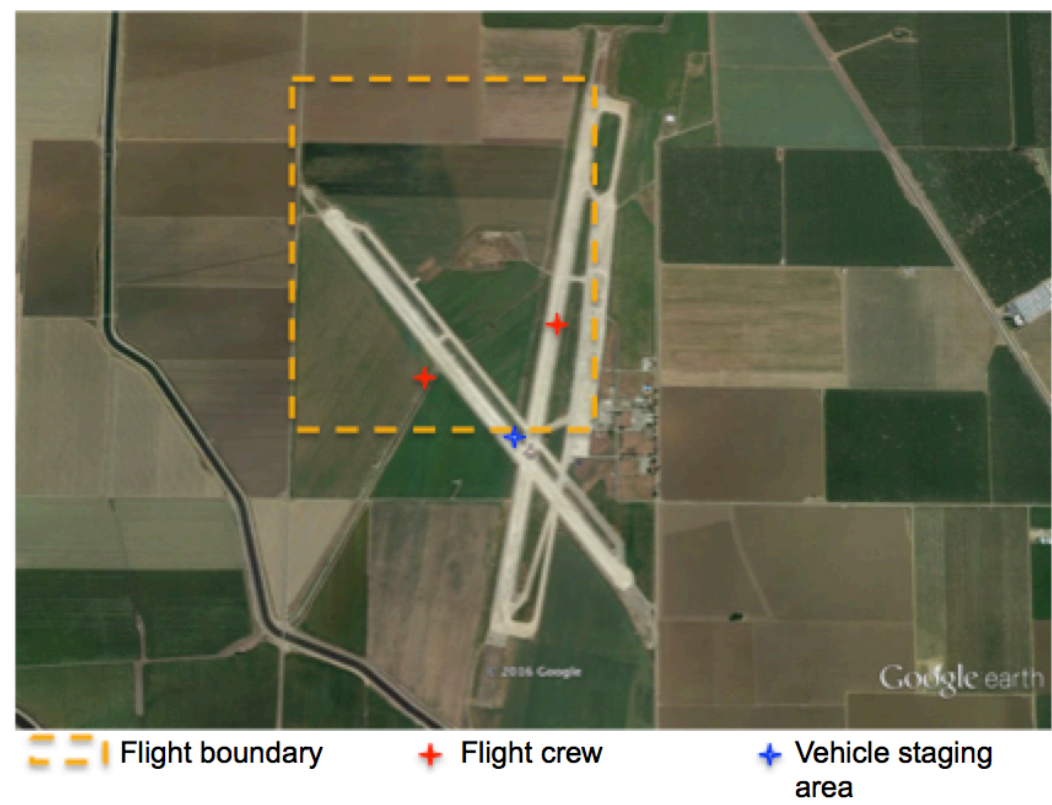

Figure 13: NASA Crows Landing Facility 


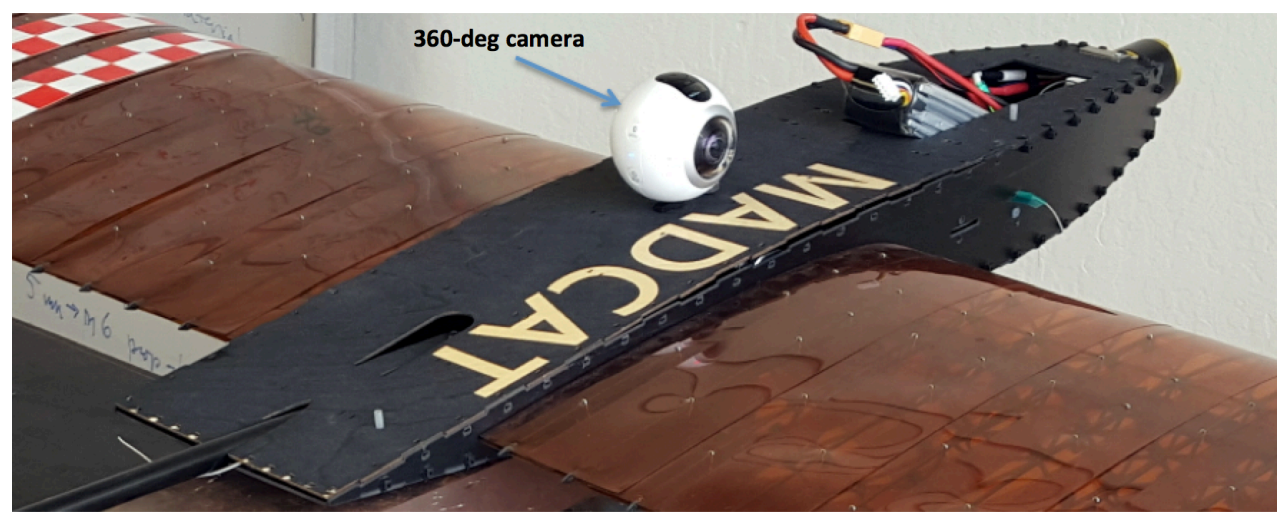

Figure 14: 360-deg camera footage in flight

\section{System Identification Results}

The measured IMU data from fuselage mounted IMU was used to perform system identification for MADCAT v0. For this purpose, the System Identification Programs for AirCraft (SIDPAC), a computational package developed by NASA Langley Research Center, was utilized for post-processing [11].

For illustration, data gathered from the roll axis maneuvers were used for roll directional identification. The commanded input is the torque tube position in degrees, the measured outputs are roll rate in degrees per second and angle-of-attack in degrees. The roll axis control derivatives are given in Table 2.

Table 2: Roll axis control derivatives

\begin{tabular}{|c|c|c|c|}
\hline Parameter & Estimate & $\mathbf{\%}$ & $\mathbf{9 5 \%} \boldsymbol{C}_{\boldsymbol{l}}$ \\
\hline $\boldsymbol{C}_{\boldsymbol{l} \alpha}$ & -0.5304 & 7.7 & {$[-0.613,-0.448]$} \\
\hline $\boldsymbol{C}_{\boldsymbol{l p}}$ & -0.322 & 7.1 & {$[-0.368,-0.276]$} \\
\hline $\boldsymbol{C}_{l \text { lwist }}$ & 0.00564 & 7.1 & {$[0.005,0.007]$} \\
\hline
\end{tabular}

The comparison between the identified roll axis model (or equation-error model) and the measured flight data is shown in Fig. 15. As can be seen, in general the identified model matches very well with the flight data, except the high frequency contents. 

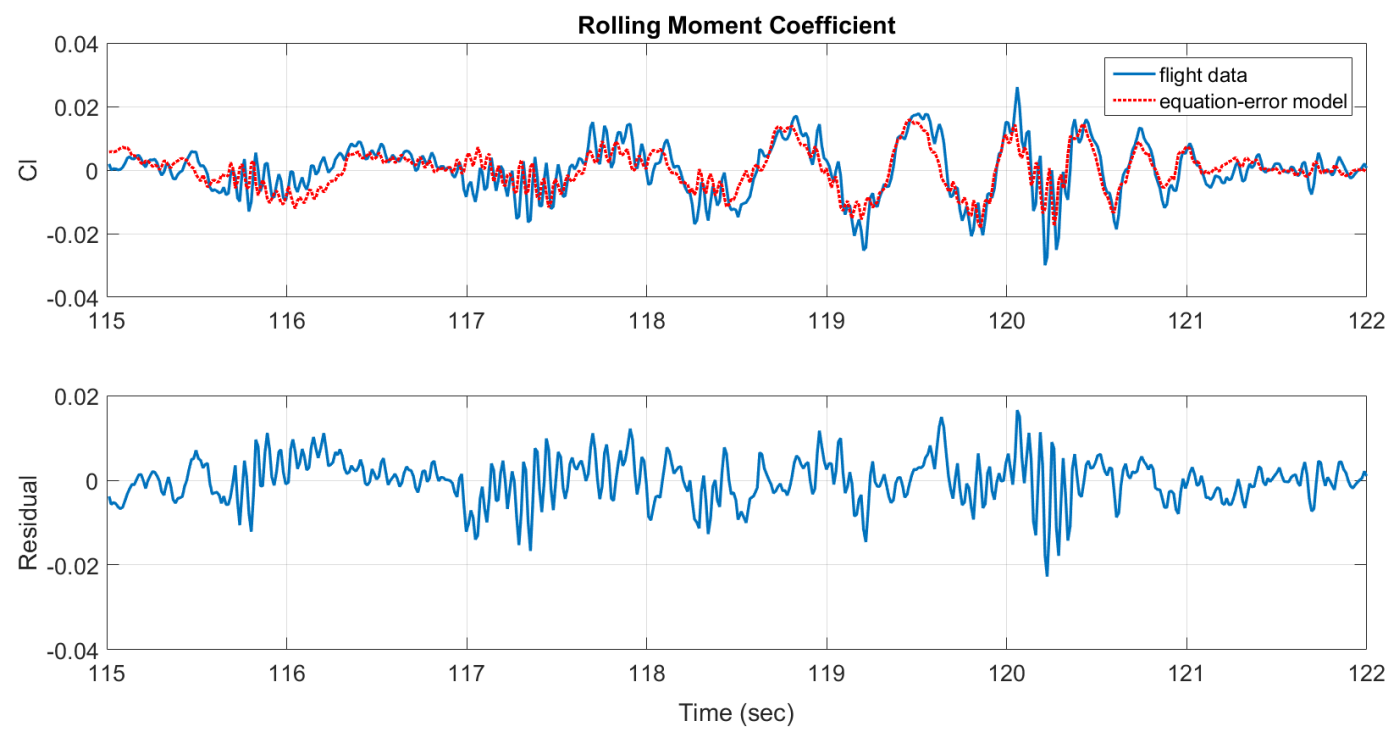

Figure 15: Rolling moment frequency sweep; identified model vs. flight data.

\section{Conclusions}

In this paper, we have developed an innovative aerostructure concept by utilizing advanced digital composite materials and discrete construction technologies. The lattice-based composite flexible wings were servo actuated and designed to perform continuous span-wise twist for ideal roll actuation. Through extensive bench tests and wind tunnel tests, the flexible wings were shown to behave as conventional wing design, but with added versatilities that would enable new mission objectives. A scaled prototype UAV MADCAT v0 was built and successfully flight tested to assess and demonstrate its maneuverability and simplified production process.

\section{Acknowledgement}

This research is funded by NASA ARMD Convergent Aeronautics Solutions (CAS) Project. The authors would like to thank Professor Neil Gershenfeld, Center for Bits and Atoms at MIT; Professor Weihua Su, University of Alabama; Professor Mircea Teodorescu, University of California at Santa Cruz; and Professor George Zhu, Michigan State University, for their helpful discussions and kind support.

\section{References}

[1] Del Rosario Project Overview, NASA FAP 2012 Technical Conference.

[2] Cheung, K. C. and Gershenfeld, N., "Reversibly assembled cellular composite materials," Science, vol. 341, No. 6151, 2013, pp. 1219-1221.

[3] Cheung, K., "Digital Cellular Solids: reconfigurable composite materials," Ph.D. Thesis, MIT, 2012. 
[4] Nguyen, N., Urnes, J., “Aeroelastic Modeling of Elastically Shaped Aircraft Concept via Wing Shaping Control for Drag Reduction," AIAA Atmospheric Flight Mechanics Conference, AIAA2012-4642, August 2012.

[5] Swei, S., Nguyen, N., "Aeroelastic Wing Shaping Control Subject to Actuation Constraints," AIAA SciTech Forum, AIAA 2014-1041, January 2014

[6] Nguyen, N., "Elastically Shaped Future Air Vehicle Concept," NASA Innovation Fund 2010.

[7] Urnes, J., Nguyen, N., Ippolito, C., Totah, J., Trinh, K., Ting, E., "A Mission-Adaptive Variable Camber Flap Control System to Optimize High Lift and Cruise Lift-to-Drag Ratios of Future $\mathrm{N}+3$ Transport Aircraft," AIAA Aerospace Sciences Meeting, AIAA 2013-0214, January 2013.

[8] Jenett, B., Calisch, S., Cellucci, D., Cramer, N., Gershenfeld, N., Swei, S., and Cheung, K., "Digital Morphing Wing: Active Wing Shaping Concept Using Composite Lattice-Based Cellular Structures," Soft Robotics (SoRo), 2016, doi:10.1089/soro.2016.0032.

doi: 10.2514/1.45452

[9] Cramer, N., Swei, S., Cheung, K. C., and Teodorescu, M., "Extended discrete-time transfer matrix approach to modeling and decentralized control of lattice-based structures," Journal of Structural Control and Health Monitoring, Vol. 23, No. 10, 2016, pp. 1256-1272.

[10] Mazhari, A., "Flight Implementation of NARI Active Wing Shaping Control Concept Using Composite Lattice-based Structures," Master Thesis, San Jose State University, December 2015. doi: $10.2514 / 2.7217$

[11] Morelli, Eugene A., "System Indentification Programs for Aircraft (SIDPAC)," AIAA Atmospheric Flight Mechanics Conference, 2002, Monterey, CA http://dx.doi.org/10.2514/6.2002-4704

[12] Cramer, Nick B., Kenneth Cheung, and Sean Shan-Min Swei. "Design and Testing of a Lattice-based Cellular Component Active Twist Wing." 24th AIAA/AHS Adaptive Structures Conference. 2016. 